\title{
A CLASS OF CONSISTENT TESTS FOR EXPONENTIALITY BASED ON THE EMPIRICAL LAPLACE TRANSFORM
}

\author{
LUDWIG BARINGHAUS AND NORBERT HENZE \\ Institut für Mathematische Stochastik, Universität Hannover, \\ Welfengarten 1, D-3000 Hannover 1, FRG
}

(Received November 6, 1989; revised May 14, 1990)

\begin{abstract}
The Laplace transform $\psi(t)=E[\exp (-t X)]$ of a random variable with exponential density $\lambda \exp (-\lambda x), x \geq 0$, satisfies the differential equation $(\lambda+t) \psi^{\prime}(t)+\psi(t)=0, t \geq 0$. We study the behaviour of a class of consistent ("omnibus") tests for exponentiality based on a suitably weighted integral of $\left[\left(\hat{\lambda}_{n}+t\right) \psi_{n}^{\prime}(t)+\psi_{n}(t)\right]^{2}$, where $\hat{\lambda}_{n}$ is the maximum-likelihood-estimate of $\lambda$ and $\psi_{n}$ is the empirical Laplace transform, each based on an i.i.d. sample $X_{1}, \ldots, X_{n}$.
\end{abstract}

Key words and phrases: Exponential distribution, goodness-of-fit test, empirical Laplace transform, consistency.

\section{Introduction}

Apart from the normal distribution, the exponential distribution is probably the most widely used probability law in statistical analysis, especially in connection with life testing and reliability theory. Therefore it is not surprising that many tests for exponentiality have been proposed in the literature (see e.g. D'Agostino and Stephens (1986) and Spurrier (1984)). Since the alternatives to the exponential distribution are rarely known in practice and the choice of a test should not be done on the basis of given data, omnibus tests for exponentiality which aim at detecting all distributional departures from exponentiality are of great importance, two prominent members of in this line of work are the tests of Cramér-von Mises and Anderson-Darling (see Davis and Stephens (1989)).

It is clear that each omnibus test for exponentiality must use some characterizing equation (property) of the class of exponential distributions and a distance statistic which measures the deviation from this equation for the empirical distribution of the observed sample.

In this paper, we study a class of omnibus tests for exponentiality based on a differential equation for the Laplace transform, characteristic for the family of exponential distributions. To be specific, let $X, X_{1}, \ldots, X_{n}$ be independent identically distributed non-negative random variables, and let $\operatorname{Exp}(\lambda)$ denote the exponential distribution with density $\lambda \exp (-\lambda x), x \geq 0$. The problem is then to 
test, on the basis of $X_{1}, \ldots, X_{n}$, the composite hypothesis

$H_{0}$ : The law of $X$ is $\operatorname{Exp}(\lambda)$ for some $\lambda>0$

against the general alternative that $X$ is not exponentially distributed. The rationale for the new test is as follows: If the distribution of $X$ is $\operatorname{Exp}(\lambda)$, the Laplace transform $\psi(t)=E[\exp (-t X)], t \geq 0$ of $X$ is given by $\psi(t)=\lambda /(\lambda+t)$ and thus, satisfies the differential equation

$$
(\lambda+t) \psi^{\prime}(t)+\psi(t)=0, \quad \forall t \geq 0
$$

subject to the boundary condition $\psi(0)=1$. Since the distribution of a nonnegative random variable is determined by its Laplace transform, the equation (1.1) characterizes the exponential distribution $\operatorname{Exp}(\lambda)$. Letting

$$
\psi_{n}(t)=\frac{1}{n} \sum_{j=1}^{n} \exp \left(-t X_{j}\right)
$$

denote the empirical Laplace transform of $X_{1}, \ldots, X_{n}$ and $\hat{\lambda}_{n}=\bar{X}_{n}^{-1}$ the maximum-likelihood-estimator of $\lambda$, where $\bar{X}_{n}=(1 / n) \sum_{j=1}^{n} X_{j}$, the test statistic proposed is the weighted integral

$$
T_{n, a}=n \int_{0}^{\infty}\left[\left(\hat{\lambda}_{n}+t\right) \psi_{n}^{\prime}(t)+\psi_{n}(t)\right]^{2} \bar{X}_{n} \exp \left(-a \bar{X}_{n} t\right) d t,
$$

where $a>0$ is a positive constant. It will be seen in Section 3 that a test for exponentiality rejecting the hypothesis $H_{0}$ for large values of $T_{n, a}$ leads to a consistent procedure for any positive $a$. However, the choice of $a$ has a pronounced influence on the power performance of the test (see Section 4).

Some motivations to consider the weight function $\bar{X}_{n} \exp \left(-\bar{X}_{n} a t\right)$ are as follows: Firstly, $T_{n, a}$ may be computed in an easy way (see below) and has the desirable feature of being scale invariant. Secondly, from Tauberian theorems on Laplace transforms (see e.g. Feller (1966), Chapter XIII.5), it is known that the tail behaviour of a probability distribution concentrated on $[0, \infty)$ is reflected by the behaviour of its Laplace transform at zero and vice versa. Consequently, choosing a small value of $a$ and thus, letting the weight function decay slowly, should result in good power properties against alternative distributions having a point mass or infinite density at zero. On the other hand, a large value of $a$ implying that the weight function puts most of its mass near zero should be a safeguard against alternative distributions with great difference in tail behaviour from the exponential distribution.

Straightforward manipulation of integrals gives the computationally simple form

$$
\begin{aligned}
T_{n, a}=\frac{1}{n} \sum_{i, j=1}^{n}[ & \frac{\left(1-Y_{i}\right)\left(1-Y_{j}\right)}{Y_{i}+Y_{j}+a}-\frac{Y_{i}+Y_{j}}{\left(Y_{i}+Y_{j}+a\right)^{2}} \\
& \left.+\frac{2 Y_{i} Y_{j}}{\left(Y_{i}+Y_{j}+a\right)^{2}}+\frac{2 Y_{i} Y_{j}}{\left(Y_{i}+Y_{j}+a\right)^{3}}\right],
\end{aligned}
$$


where $Y_{j}=X_{j} / \bar{X}_{n}, 1 \leq j \leq n$. This shows that $T_{n, a}=n V_{n}\left(\hat{\lambda}_{n}\right)$, where

$$
V_{n}(\lambda)=\frac{1}{n^{2}} \sum_{i, j=1}^{n} h_{a}\left(X_{i}, X_{j} ; \lambda\right)
$$

is a degree-two $V$-statistic with kernel

$$
\begin{aligned}
h_{a}(x, y ; \lambda)= & \frac{(1-\lambda x)(1-\lambda y)}{\lambda(x+y)+a}-\frac{\lambda(x+y)}{(\lambda(x+y)+a)^{2}} \\
& +\frac{2 \lambda^{2} x y}{(\lambda(x+y)+a)^{2}}+\frac{2 \lambda^{2} x y}{(\lambda(x+y)+a)^{3}}
\end{aligned}
$$

(see, e.g. Serfling (1980)). Moreover, the distribution of $T_{n, a}$ under $H_{0}$ is seen to be independent of the underlying parameter $\lambda$. An alternative expression of $T_{n, a}$ is

$$
T_{n, a}=\int_{0}^{1} Z_{n}^{2}(u) u^{a-1} d u
$$

where

$$
Z_{n}(u)=n^{-1 / 2} \sum_{j=1}^{n} u^{Y_{j}}\left[1-(1-\log (u)) Y_{j}\right], \quad 0 \leq u \leq 1
$$

The representation (1.2) in terms of a functional of a stochastic process is particularly useful for deriving the asymptotic null distribution of $T_{n, a}$ as $n \rightarrow \infty$. This will be done in the next section.

\section{The limiting null distribution of the test statistic}

The stochastic process $Z_{n}$ introduced in (1.3) may be regarded as a random element in $C[0,1]$, the Banach space of real valued continuous functions on the unit interval, endowed with the supremum norm $\sup _{0 \leq u \leq 1}|x(u)|, x \in C[0,1]$. Obviously, $T_{n, a}$ is a continuous function of $Z_{n}$. We shall prove that, under the hypothesis $H_{0}$ of exponentiality, $Z_{n}$ tends in distribution to a zero-mean Gaussian process $Z=\{Z(u), 0 \leq u \leq 1\}$ with continuous sample paths. Consequently, the limiting null distribution of $T_{n, a}$ is the same as that of

$$
T_{a}=\int_{0}^{1} Z(u)^{2} u^{a-1} d u
$$

It is well known that the distribution of $T_{a}$ is that of $\sum_{j \geq 1} \gamma_{j} N_{j}^{2}$, where $N_{1}, N_{2}, \ldots$ are independent unit normal random variables, and $\gamma_{j}, j \geq 1$, are the eigenvalues of the integral operator associated with the kernel $k(u, v)=\operatorname{Cov}(Z(u), Z(v))$, $0 \leq u, v \leq 1$, i.e.

$$
\gamma_{j} \varphi_{j}(u)=\int_{0}^{1} k(u, v) \varphi_{j}(v) v^{a-1} d v, \quad 0 \leq u \leq 1
$$


The eigenfunction $\varphi_{j}$ corresponding to $\gamma_{j}$ is square integrable with respect to the measure $d \nu_{a}(u)=u^{a-1} d u$ on the unit interval. The kernel $k(u, v)$ turns out to be

$$
k(u, v)=\frac{(1-\log u)(1-\log v)+(\log u)(\log v)}{(1-\log (v u))^{3}}-\frac{1}{(1-\log u)^{2}(1-\log v)^{2}}
$$

$(0 \leq u, v \leq 1)$. In what follows, we may assume that the random variables $X_{j}$ are exponentially distributed with the parameter $\lambda=1$. The stochastic process $W_{n}=\left\{W_{n}(u), 0 \leq u \leq 1\right\}$, where

$$
W_{n}(u)=n^{-1 / 2} \sum_{j=1}^{n}\left[u^{X_{j}}\left(1-(1-\log u) X_{j}\right)+\frac{X_{j}-1}{(1-\log u)^{2}}\right], \quad 0 \leq u \leq 1,
$$

can also be regarded as a random element of $C[0,1]$. We first show that $W_{n}$ converges in distribution to $Z$. By applying the multivariate central limit theorem we see that the finite dimensional distributions of $W_{n}$ converge weakly to multivariate normal distributions with zero means and covariance matrices determined by the kernel $k(\cdot, \cdot)$ given in $(2.1)$. Now,

$$
e(u, v)=\left|(1-\log u)^{-1 / 4}-(1-\log v)^{-1 / 4}\right|, \quad 0 \leq u, v \leq 1,
$$

defines a continuous metric $e$ on the unit interval satisfying the metric entropy condition

$$
\int_{0}^{1}(\log N(u))^{1 / 2} d u<\infty .
$$

Here, for each $u>0, N(u)$ is the smallest positive integer $m$ such that the unit interval can be covered by $m$ subsets, each having a diameter at most $2 u$ with respect to $e$. Letting

$$
S(u)=u^{X_{1}}\left(1-(1-\log u) X_{1}\right)+\frac{X_{1}-1}{(1-\log u)^{2}}, \quad 0 \leq u \leq 1,
$$

the second mean value theorem implies that there is a positive constant $c$ such that

$$
|S(u)-S(v)| \leq c \max \left(X_{1}^{2}, X_{1}^{-1 / 4}\right) e(u, v), \quad 0 \leq u, v \leq 1 .
$$

Since $E\left[\max \left(X_{1}^{2}, X_{1}^{-1 / 4}\right)^{2}\right]<\infty$, the sequence of distributions of $W_{n}$ in $C[0,1]$ is tight and converges weakly to the distribution of a zero-mean Gaussian process with continuous sample paths and covariance function $k(\cdot, \cdot)$ (see, e.g. Araujo and Giné (1980)).

Introducing for $\xi>0$, the process $M_{n}(\xi)=\left\{M_{n}(\xi, u), 0 \leq u \leq 1\right\}$, where

$$
M_{n}(\xi, u)=n^{-1 / 2} \sum_{j=1}^{n} u^{\xi X_{j}}\left(1-(1-\log u) \xi X_{j}\right), \quad 0 \leq u \leq 1,
$$


and denoting by $L_{n}$ and $\hat{L}_{n}$ the processes

$$
L_{n}(u)=(1-\log u)^{-2} n^{-1 / 2} \sum_{j=1}^{n}\left(X_{j}-1\right), \quad 0 \leq u \leq 1
$$

and

$$
\hat{L}_{n}(u)=-(1-\log u)^{-2} n^{1 / 2}\left(\hat{\lambda}_{n}-1\right), \quad 0 \leq u \leq 1
$$

we have that

$$
\left\|Z_{n}-W_{n}\right\|_{a}=\left\|M_{n}\left(\hat{\lambda}_{n}\right)-M_{n}(1)-L_{n}\right\|_{a}
$$

where, generically,

$$
\|f\|_{a}^{2}=\int_{0}^{1} f^{2} d \nu_{a}, \quad f \text { square integrable with respect to } d \nu_{a} .
$$

Using a Taylor expansion of $M_{n}(\xi, 1)$ in a neighbourhood of $\xi=1$ we see that $\left\|M_{n}\left(\hat{\lambda}_{n}\right)-M_{n}(1)-\hat{L}_{n}\right\|_{a}=o_{P}(1)$. Since $\left\|L_{n}-\hat{L}_{n}\right\|_{a}=o_{P}(1)$ it follows that $\left\|Z_{n}-W_{n}\right\|_{a}=o_{P}(1)$. Summarizing, we have the following result.

THEOREM 2.1. The limiting null distribution of the test statistic $T_{n, a}$ is that of $\sum_{j \geq 1} \gamma_{j} N_{j}^{2}$, where $N_{1}, N_{2}, \ldots$ are independent unit normal random variables, and $\gamma_{1}, \gamma_{2}, \ldots$ are the eigenvalues of the integral operator associated with the kernel $k(\cdot, \cdot)$ given in $(2.1)$.

It should be remarked that a different method of proof of the result stated above is provided by the work of De Wet and Randles (1987).

\section{Consistency}

For a given level of significance $\alpha \in(0,1)$, let $t_{n, a}(\alpha)$ be the $(1-\alpha)$-quantile of $T_{n, a}$ when the hypothesis $H_{0}$ is true.

THEOREM 3.1. The test rejecting the hypothesis of exponentiality if $T_{n, a}>$ $t_{n, a}(\alpha)$ is consistent against any fixed non-exponential distribution having finite positive first moment.

Proof. Let $X_{1}$ have a distribution with finite expectation $\lambda>0$ and Laplace-transform $\phi(t), t \geq 0$. Then $n^{-1} T_{n, a}$ tends to

$$
\lambda \int_{0}^{\infty}\left(\left(\lambda^{-1}+t\right) \phi^{\prime}(t)+\phi(t)\right)^{2} \exp (-a \lambda t) d t
$$

in probability. This stochastic limit is zero if and only if $\phi$ is the Laplacetransform of an exponential distribution. Thus, for non-exponential distributions, $\lim _{n \rightarrow \infty} P\left(T_{n, a} \leq t_{n, a}(\alpha)\right)=0$. 


\section{Power results}

The main justification to propose a new test is that it provides a higher power than the presently used procedures. To compare the power of the proposed test with some of the prominent competitive procedures, especially the omnibus tests of Cramér-von Mises and Anderson-Darling, a Monte Carlo simulation study was done.

The following procedures were compared.

(i) The new test based on $T_{n, a}$ for $a=0.1, a=1$ and $a=10$ which is indicated as $T(.1), T(1)$ and $T(10)$ in Tables 4 and 5. Critical points for $T_{n, a}$ may be obtained from Table $1(a=0.1)$, Table $2(a=1)$ and Table $3(a=10)$. The entries in Tables 1-3 represent 20\%-trimmed means of 10 Monte Carlo estimates, each based on 10000 replications. The results indicate rapid convergence of the true quantiles to their limiting values as $n \rightarrow \infty$.

Table 1. Empirical percentage points for $T_{n, a}, a=0.1$.

\begin{tabular}{rcccccc}
\hline & \multicolumn{6}{c}{$1-\alpha$} \\
$n$ & 0.5 & 0.75 & 0.9 & 0.95 & 0.975 & 0.99 \\
\hline 5 & 0.284 & 0.534 & 0.832 & 1.071 & 1.529 & 2.465 \\
6 & 0.290 & 0.543 & 0.854 & 1.091 & 1.569 & 2.437 \\
7 & 0.293 & 0.546 & 0.871 & 1.130 & 1.585 & 2.414 \\
8 & 0.293 & 0.548 & 0.887 & 1.181 & 1.601 & 2.397 \\
9 & 0.294 & 0.550 & 0.908 & 1.212 & 1.614 & 2.386 \\
10 & 0.294 & 0.553 & 0.923 & 1.231 & 1.624 & 2.376 \\
11 & 0.294 & 0.556 & 0.936 & 1.246 & 1.632 & 2.367 \\
12 & 0.294 & 0.560 & 0.946 & 1.258 & 1.639 & 2.359 \\
13 & 0.294 & 0.563 & 0.952 & 1.268 & 1.646 & 2.351 \\
14 & 0.294 & 0.565 & 0.957 & 1.277 & 1.653 & 2.344 \\
15 & 0.294 & 0.567 & 0.961 & 1.284 & 1.659 & 2.336 \\
16 & 0.294 & 0.569 & 0.964 & 1.290 & 1.666 & 2.330 \\
17 & 0.294 & 0.571 & 0.967 & 1.295 & 1.672 & 2.325 \\
18 & 0.294 & 0.572 & 0.970 & 1.300 & 1.678 & 2.320 \\
19 & 0.294 & 0.573 & 0.973 & 1.305 & 1.684 & 2.316 \\
20 & 0.294 & 0.574 & 0.975 & 1.309 & 1.689 & 2.312 \\
25 & 0.294 & 0.576 & 0.986 & 1.327 & 1.707 & 2.305 \\
30 & 0.294 & 0.578 & 0.994 & 1.339 & 1.722 & 2.301 \\
35 & 0.294 & 0.579 & 1.001 & 1.346 & 1.731 & 2.298 \\
40 & 0.294 & 0.580 & 1.003 & 1.351 & 1.735 & 2.295 \\
45 & 0.294 & 0.580 & 1.005 & 1.354 & 1.737 & 2.292 \\
50 & 0.294 & 0.580 & 1.006 & 1.356 & 1.739 & 2.289 \\
\hline
\end{tabular}


Table 2. Empirical percentage points for $T_{n, a}, a=1$.

\begin{tabular}{rcccccc}
\hline & \multicolumn{6}{c}{$1-\alpha$} \\
$n$ & 0.5 & 0.75 & 0.9 & 0.95 & 0.975 & 0.99 \\
\hline 5 & 0.050 & 0.118 & 0.205 & 0.262 & 0.308 & 0.378 \\
6 & 0.049 & 0.117 & 0.209 & 0.272 & 0.329 & 0.407 \\
7 & 0.049 & 0.117 & 0.211 & 0.276 & 0.338 & 0.425 \\
8 & 0.049 & 0.117 & 0.213 & 0.282 & 0.352 & 0.446 \\
9 & 0.048 & 0.117 & 0.214 & 0.286 & 0.359 & 0.464 \\
10 & 0.048 & 0.117 & 0.215 & 0.289 & 0.366 & 0.469 \\
11 & 0.048 & 0.116 & 0.216 & 0.291 & 0.369 & 0.476 \\
12 & 0.048 & 0.116 & 0.217 & 0.294 & 0.372 & 0.482 \\
13 & 0.047 & 0.116 & 0.217 & 0.295 & 0.374 & 0.486 \\
14 & 0.047 & 0.116 & 0.217 & 0.296 & 0.376 & 0.490 \\
15 & 0.047 & 0.116 & 0.217 & 0.297 & 0.378 & 0.492 \\
16 & 0.047 & 0.116 & 0.217 & 0.298 & 0.380 & 0.495 \\
17 & 0.047 & 0.116 & 0.218 & 0.299 & 0.381 & 0.497 \\
18 & 0.047 & 0.115 & 0.218 & 0.300 & 0.383 & 0.499 \\
19 & 0.047 & 0.115 & 0.219 & 0.302 & 0.386 & 0.501 \\
20 & 0.047 & 0.115 & 0.220 & 0.303 & 0.389 & 0.506 \\
25 & 0.047 & 0.115 & 0.220 & 0.304 & 0.392 & 0.512 \\
30 & 0.047 & 0.115 & 0.221 & 0.305 & 0.393 & 0.513 \\
35 & 0.047 & 0.115 & 0.221 & 0.306 & 0.396 & 0.515 \\
40 & 0.047 & 0.114 & 0.222 & 0.308 & 0.398 & 0.521 \\
45 & 0.047 & 0.114 & 0.223 & 0.309 & 0.399 & 0.523 \\
50 & 0.047 & 0.114 & 0.223 & 0.310 & 0.401 & 0.526 \\
\hline
\end{tabular}

(ii) The tests of Cramér-von Mises and Anderson-Darling. These are based on measures of discrepancy between the empirical distribution function

$$
G_{n}(u)=\frac{1}{n} \sum_{j=1}^{n} I\left\{W_{(j)} \leq u\right\}
$$

of $W_{(j)}=1-\exp \left(-Y_{(j)}\right), 1 \leq j \leq n$, and the uniform distribution function on the unit interval. Here and in what follows, $Y_{(1)} \leq \cdots \leq Y_{(n)}$ denote the order statistics of $Y_{1}, \ldots, Y_{n}$, and $I\{A\}$ is the indicator function of an event $A$. The Cramér-von Mises statistic is

$$
\begin{aligned}
C^{2} & =n \int_{0}^{1}\left(G_{n}(u)-u\right)^{2} d u \\
& =\sum_{j=1}^{n}\left(W_{(j)}-\frac{2 j-1}{2 n}\right)^{2}+\frac{1}{12 n}
\end{aligned}
$$


Table 3. Empirical percentage points for $T_{n, a}, a=10$.

\begin{tabular}{rcccccc}
\hline & \multicolumn{6}{c}{$1-\alpha$} \\
\cline { 2 - 7 }$n$ & 0.5 & 0.75 & 0.9 & 0.95 & 0.975 & 0.99 \\
\hline 5 & 0.0010 & 0.0022 & 0.0035 & 0.0043 & 0.0050 & 0.0073 \\
6 & 0.0010 & 0.0023 & 0.0038 & 0.0047 & 0.0056 & 0.0089 \\
7 & 0.0010 & 0.0024 & 0.0040 & 0.0050 & 0.0062 & 0.0100 \\
8 & 0.0010 & 0.0025 & 0.0041 & 0.0053 & 0.0067 & 0.0109 \\
9 & 0.0010 & 0.0025 & 0.0043 & 0.0056 & 0.0072 & 0.0121 \\
10 & 0.0010 & 0.0025 & 0.0044 & 0.0058 & 0.0076 & 0.0124 \\
11 & 0.0010 & 0.0026 & 0.0045 & 0.0060 & 0.0079 & 0.0127 \\
12 & 0.0010 & 0.0026 & 0.0046 & 0.0062 & 0.0081 & 0.0129 \\
13 & 0.0010 & 0.0026 & 0.0047 & 0.0063 & 0.0084 & 0.0131 \\
14 & 0.0010 & 0.0026 & 0.0048 & 0.0065 & 0.0086 & 0.0134 \\
15 & 0.0010 & 0.0027 & 0.0049 & 0.0066 & 0.0088 & 0.0139 \\
16 & 0.0010 & 0.0027 & 0.0049 & 0.0067 & 0.0090 & 0.0141 \\
17 & 0.0011 & 0.0027 & 0.0050 & 0.0068 & 0.0091 & 0.0143 \\
18 & 0.0011 & 0.0027 & 0.0050 & 0.0069 & 0.0093 & 0.0145 \\
19 & 0.0011 & 0.0028 & 0.0051 & 0.0070 & 0.0095 & 0.0147 \\
20 & 0.0011 & 0.0028 & 0.0052 & 0.0072 & 0.0097 & 0.0149 \\
25 & 0.0011 & 0.0028 & 0.0054 & 0.0074 & 0.0100 & 0.0152 \\
30 & 0.0011 & 0.0029 & 0.0055 & 0.0077 & 0.0103 & 0.0153 \\
35 & 0.0011 & 0.0029 & 0.0056 & 0.0079 & 0.0105 & 0.0153 \\
40 & 0.0011 & 0.0029 & 0.0057 & 0.0080 & 0.0106 & 0.0154 \\
45 & 0.0011 & 0.0030 & 0.0058 & 0.0081 & 0.0107 & 0.0154 \\
50 & 0.0011 & 0.0030 & 0.0058 & 0.0082 & 0.0108 & 0.0155 \\
\hline & & & & & &
\end{tabular}

whereas the Anderson-Darling procedure is based on

$$
\begin{aligned}
A^{2} & =n \int_{0}^{1} \frac{\left(G_{n}(u)-u\right)^{2}}{u(1-u)} d u \\
& =-n-\frac{1}{n} \sum_{j=1}^{n}(2 j-1)\left[\log \left(W_{(j)}\right)+\log \left(1-W_{(n-j+1)}\right)\right] .
\end{aligned}
$$

The tests were carried out using the modifications and percentage points given in Table 4.11 of D'Agostino and Stephens (1986). The Anderson-Darling test should be used with caution due to severe effects of recording errors close to zero.

(iii) The test of Moran (Moran (1951)). This procedure is based on the statistic

$$
M=-2 \sum_{j=1}^{n} \log \left(Y_{j}\right)
$$


Table 4. Percentage of 5000 Monte Carlo samples declared significant by the various tests of exponentiality; test size $\alpha=0.05$; sample size $n=20$.

\begin{tabular}{|c|c|c|c|c|c|c|c|c|c|}
\hline Alternative & $T(.1)$ & $T(1)$ & $T(10)$ & $M$ & $Q_{1}$ & $W^{*}$ & $C^{2}$ & $A^{2}$ & $S$ \\
\hline $\operatorname{Gamma}(0.4)$ & 91 & 82 & 67 & 92 & 40 & 52 & 75 & 89 & 18 \\
\hline $\operatorname{Gamma}(0.6)$ & 53 & 39 & 31 & 52 & 16 & 20 & 32 & 48 & 9 \\
\hline $\operatorname{Gamma}(0.8)$ & 17 & 12 & 11 & 16 & 8 & 8 & 10 & 15 & 6 \\
\hline $\operatorname{Gamma}(1.0)$ & 5 & 5 & 5 & 5 & 5 & 5 & 5 & 5 & 6 \\
\hline $\operatorname{Gamma}(1.4)$ & 11 & 16 & 10 & 17 & 6 & 13 & 15 & 13 & 5 \\
\hline $\operatorname{Gamma}(1.6)$ & 19 & 26 & 17 & 28 & 7 & 21 & 24 & 21 & 6 \\
\hline $\operatorname{Gamma}(1.8)$ & 32 & 41 & 27 & 43 & 12 & 32 & 36 & 34 & 8 \\
\hline $\operatorname{Gamma}(2.0)$ & 44 & 53 & 38 & 57 & 15 & 43 & 49 & 46 & 7 \\
\hline $\operatorname{Gamma}(2.4)$ & 67 & 75 & 56 & 78 & 27 & 61 & 69 & 68 & 10 \\
\hline $\operatorname{Gamma}(3.0)$ & 89 & 93 & 80 & 94 & 46 & 82 & 89 & 88 & 12 \\
\hline Weibull(0.4) & $*$ & 99 & 97 & * & 83 & 92 & 98 & * & 60 \\
\hline Weibull(0.6) & 83 & 76 & 67 & 82 & 40 & 54 & 69 & 81 & 25 \\
\hline Weibull(0.8) & 29 & 25 & 24 & 27 & 12 & 18 & 20 & 28 & 11 \\
\hline Weibull(1.2) & 10 & 14 & 10 & 14 & 6 & 13 & 14 & 12 & 6 \\
\hline Weibull(1.4) & 26 & 38 & 28 & 38 & 10 & 33 & 34 & 31 & 8 \\
\hline Weibull(1.6) & 51 & 67 & 54 & 65 & 20 & 61 & 62 & 59 & 14 \\
\hline Weibull(2.0) & 88 & 96 & 92 & 95 & 47 & 93 & 93 & 92 & 26 \\
\hline Uniform $(0,1)$ & 33 & 60 & 66 & 45 & 27 & 77 & 67 & 63 & 79 \\
\hline Half-Normal & 11 & 20 & 17 & 18 & 9 & 23 & 21 & 17 & 11 \\
\hline Half-Cauchy & 58 & 65 & 70 & 55 & 53 & 67 & 64 & 64 & 57 \\
\hline Log-Normal(0.5) & $*$ & 99 & 91 & $*$ & 87 & 91 & 99 & 99 & 9 \\
\hline Log-Normal(0.7) & 67 & 61 & 37 & 70 & 30 & 39 & 61 & 62 & 9 \\
\hline Log-Normal $(0.8)$ & 37 & 34 & 18 & 42 & 16 & 21 & 34 & 35 & 11 \\
\hline Log-Normal(1.0) & 9 & 12 & 17 & 8 & 11 & 17 & 16 & 15 & 18 \\
\hline Log-Normal(1.5) & 56 & 64 & 67 & 48 & 44 & 60 & 61 & 63 & 43 \\
\hline$\chi_{1}^{2}$ & 76 & 62 & 49 & 76 & 25 & 34 & 53 & 71 & 13 \\
\hline Power(0.5) & 99 & * & * & * & 87 & * & * & * & 98 \\
\hline Power(0.8) & 67 & 88 & 91 & 77 & 43 & 96 & 91 & 90 & 90 \\
\hline Power(1.2) & 16 & 32 & 38 & 21 & 20 & 50 & 42 & 38 & 65 \\
\hline Power(1.4) & 11 & 14 & 18 & 12 & 18 & 27 & 24 & 24 & 51 \\
\hline Power(2.0) & 45 & 14 & 3 & 40 & 21 & 3 & 19 & 41 & 23 \\
\hline Power(3.0) & 90 & 65 & 28 & 90 & 38 & 11 & 63 & 88 & 9 \\
\hline Power(4.0) & 99 & 91 & 64 & 99 & 58 & 39 & 89 & 98 & 9 \\
\hline $\operatorname{JSHAPE}(0.5)$ & 39 & 45 & 51 & 36 & 29 & 44 & 41 & 44 & 33 \\
\hline JSHAPE(1.0) & 79 & 83 & 84 & 77 & 65 & 79 & 80 & 82 & 62 \\
\hline JSHAPE(1.5) & 95 & 95 & 95 & 94 & 85 & 92 & 94 & 95 & 79 \\
\hline $\operatorname{LIFR}(1.0)$ & 10 & 18 & 13 & 15 & 8 & 19 & 18 & 14 & 9 \\
\hline $\operatorname{LIFR}(2.0)$ & 17 & 28 & 23 & 25 & 11 & 30 & 29 & 25 & 13 \\
\hline $\operatorname{LIFR}(4.0)$ & 26 & 42 & 36 & 37 & 14 & 44 & 44 & 37 & 15 \\
\hline $\operatorname{LIFR(6.0)}$ & 31 & 49 & 43 & 43 & 17 & 52 & 49 & 44 & 18 \\
\hline $\operatorname{LIFR}(10.0)$ & 40 & 59 & 53 & 51 & 21 & 62 & 58 & 54 & 20 \\
\hline
\end{tabular}


Table 5. Percentage of 5000 Monte Carlo samples declared significant by the various tests of exponentiality; test size $\alpha=0.05$; sample size $n=50$.

\begin{tabular}{|c|c|c|c|c|c|c|c|c|c|}
\hline Alternative & $T(.1)$ & $T(1)$ & $T(10)$ & $M$ & $Q_{1}$ & $W^{*}$ & $C^{2}$ & $A^{2}$ & $S$ \\
\hline $\operatorname{Gamma}(0.4)$ & * & 99 & 96 & * & 64 & 87 & 99 & $*$ & 29 \\
\hline $\operatorname{Gamma}(0.6)$ & 84 & 75 & 59 & 86 & 23 & 42 & 66 & 80 & 12 \\
\hline Gamma(0.8) & 28 & 21 & 17 & 27 & 9 & 11 & 17 & 24 & 7 \\
\hline $\operatorname{Gamma}(1.0)$ & 5 & 5 & 5 & 5 & 5 & 5 & 4 & 5 & 5 \\
\hline Gamma(1.4) & 34 & 37 & 24 & 41 & 9 & 26 & 32 & 32 & 4 \\
\hline Gamma(1.6) & 62 & 64 & 44 & 69 & 17 & 45 & 56 & 58 & 6 \\
\hline $\operatorname{Gamma}(1.8)$ & 83 & 84 & 65 & 88 & 27 & 64 & 77 & 80 & 7 \\
\hline $\operatorname{Gamma}(2.0)$ & 94 & 94 & 81 & 96 & 41 & 79 & 91 & 93 & 8 \\
\hline $\operatorname{Gamma}(2.4)$ & * & 99 & 96 & $*$ & 65 & 94 & 99 & 99 & 12 \\
\hline $\operatorname{Gamma}(3.0)$ & * & $*$ & $*$ & $*$ & 89 & 99 & * & $*$ & 16 \\
\hline Weibull(0.6) & 99 & 99 & 96 & 99 & 65 & 90 & 98 & 99 & 47 \\
\hline Weibull(0.8) & 54 & 50 & 45 & 54 & 16 & 33 & 43 & 51 & 15 \\
\hline Weibull(1.2) & 25 & 32 & 24 & 32 & 8 & 26 & 28 & 27 & 5 \\
\hline Weibull(1.4) & 73 & 82 & 73 & 81 & 23 & 73 & 75 & 76 & 11 \\
\hline Weibull(1.6) & 96 & 98 & 96 & 98 & 47 & 96 & 96 & 97 & 22 \\
\hline Uniform $(0,1)$ & 79 & 96 & 99 & 80 & 52 & $*$ & 98 & 99 & $*$ \\
\hline Half-Normal & 30 & 49 & 50 & 37 & 13 & 56 & 48 & 44 & 17 \\
\hline Half-Cauchy & 88 & 93 & 95 & 86 & 83 & 94 & 93 & 92 & 88 \\
\hline Log-Normal(0.7) & $*$ & 96 & 63 & 99 & 83 & 57 & 98 & 99 & 13 \\
\hline Log-Normal(0.8) & 94 & 67 & 30 & 81 & 52 & 29 & 76 & 85 & 18 \\
\hline Log-Normal(1.0) & 34 & 18 & 28 & 15 & 23 & 29 & 30 & 34 & 34 \\
\hline Log-Normal(1.2) & 38 & 52 & 66 & 21 & 36 & 62 & 57 & 55 & 52 \\
\hline Log-Normal(1.5) & 89 & 94 & 95 & 84 & 70 & 92 & 94 & 93 & 74 \\
\hline$\chi_{1}^{2}$ & 97 & 94 & 82 & 98 & 39 & 66 & 90 & 96 & 18 \\
\hline Power(0.8) & 99 & $*$ & $*$ & 99 & 79 & * & $*$ & $*$ & * \\
\hline Power(1.2) & 42 & 72 & 88 & 39 & 35 & 94 & 86 & 86 & 99 \\
\hline Power(1.4) & 22 & 34 & 57 & 13 & 30 & 72 & 61 & 65 & 96 \\
\hline $\operatorname{Power}(2.0)$ & 74 & 25 & 3 & 64 & 34 & 6 & 49 & 76 & 68 \\
\hline $\operatorname{Power}(3.0)$ & $*$ & 95 & 48 & $*$ & 64 & 15 & 96 & * & 27 \\
\hline JSHAPE $(0.1)$ & 9 & 11 & 16 & 8 & 7 & 13 & 9 & 10 & 12 \\
\hline JSHAPE $(0.2)$ & 19 & 25 & 33 & 18 & 13 & 29 & 22 & 23 & 23 \\
\hline JSHAPE $(0.5)$ & 69 & 79 & 82 & 68 & 51 & 78 & 75 & 76 & 60 \\
\hline JSHAPE(1.0) & 98 & 99 & 99 & 98 & 92 & 99 & 99 & 99 & 92 \\
\hline $\operatorname{LIFR(1.0)}$ & 26 & 43 & 42 & 34 & 11 & 47 & 41 & 38 & 13 \\
\hline $\operatorname{LIFR(2.0)}$ & 46 & 66 & 66 & 53 & 18 & 71 & 64 & 61 & 21 \\
\hline $\operatorname{LIFR(4.0)}$ & 68 & 85 & 86 & 72 & 27 & 89 & 83 & 81 & 30 \\
\hline $\operatorname{LIFR}(6.0)$ & 78 & 92 & 92 & 81 & 34 & 94 & 91 & 89 & 34 \\
\hline $\operatorname{LIFR}(8,0)$ & 84 & 95 & 95 & 86 & 40 & 96 & 93 & 93 & 37 \\
\hline $\operatorname{LIFR}(10.0)$ & 87 & 96 & 97 & 88 & 41 & 97 & 96 & 95 & 39 \\
\hline
\end{tabular}


A two-sided test, based on $M$, is a uniformly most powerful unbiased test against Gamma alternatives (see also Shorack (1972)). Bartholomew (1957) showed it to be a strong test against Weibull alternatives. For our simulation study, critical values for $M$ (two-sided rejection region) were found for sample sizes of $n=20$ and $n=50$ by extensive simulations ( $10^{6}$ replications). A severe deficiency of the test based on $M$, is the effect on $M$ of inaccurate measurements of the values of $X_{j}$ close to zero.

(iv) The $W^{*}$-test. This test was originally proposed by Shapiro and Wilk (1972) to test the more general hypothesis

$$
\tilde{H}_{0}: P(X \geq t)=\exp (-\lambda(t-\theta)), \quad t \geq \theta, \text { for some } \lambda, \theta(\lambda>0) .
$$

It was modified by Stephens (1978) to test the hypothesis $H_{0}$ of exponentiality with origin $(=0)$ known. The test statistic is

$$
W^{*}=\frac{\left(\sum_{j=1}^{n} X_{j}\right)^{2}}{n\left[(n+1) \sum_{j=1}^{n} X_{j}^{2}-\left(\sum_{j=1}^{n} X_{j}\right)^{2}\right]}
$$

As a general test for exponentiality a two-sided rejection region must be used. Since

$$
\begin{aligned}
\frac{1}{W^{*}} & =1+(n+1) \frac{S_{n}^{2}}{\bar{X}_{n}^{2}} \\
& =1+\frac{n+1}{n} \sum_{j=1}^{n}\left(Y_{j}-1\right)^{2}
\end{aligned}
$$

with $S_{n}^{2}=n^{-1} \sum_{j=1}^{n}\left(X_{j}-\bar{X}_{n}\right)^{2}$, we see that the test merely aims at investigating the first two moments of the underlying distribution and thus, it is not an omnibus procedure. Since $W^{*}$ has the same null distribution as the statistic $W_{E}$ of Shapiro and Wilk (1972) for a sample of size $n+1$, their tables may be used to obtain critical points.

(v) The $Q_{1}$-test. This test was recently proposed by Patwardhan (1988) as a (purportedly) omnibus procedure for assessing exponentiality. It rejects the hypothesis $H_{0}$ of exponentiality for large values of

$$
Q_{1}=\left(Y_{()}-\delta\right)^{\prime} \Sigma_{()}^{-}\left(Y_{()}-\delta\right)+(n+1),
$$

where

$$
\begin{aligned}
& Y_{()}=\left(Y_{(1)}, \ldots, Y_{(n)}\right)^{\prime}, \quad Y_{(j)}=X_{(j)} / \bar{X}_{n} \quad(1 \leq j \leq n), \\
& \delta=\left(\delta_{1}, \ldots, \delta_{n}\right)^{\prime}, \quad \delta_{j}=\sum_{k=1}^{j} \frac{1}{n-k+1}
\end{aligned}
$$

and $\Sigma_{(0}^{-}$is a generalized inverse of the covariance matrix of $Y_{()}$for which an explicit expression is given by Patwardhan (1988). In the notation given above, $c^{\prime}$ 
generically denotes the transpose of a column vector $c$. Since $E\left[Y_{()}\right]=\delta$, the quadratic form occuring in (4.1) represents a standardized deviation of a plot of $Y_{(j)}$ versus $\delta_{j}(j=1, \ldots, n)$ from a straight line. An alternative expression for $Q_{1}$ is (Patwardhan (1988))

$$
Q_{1}=\frac{n+1}{n} \sum_{j=1}^{n} U_{n, j}^{2},
$$

where $U_{n, j}$ denotes the normalized scaled spacing

$$
U_{n, j}=(n-j+1)\left(Y_{(j)}-Y_{(j-1)}\right) \quad\left(j=1, \ldots, n, Y_{(0)}:=0\right)
$$

Although a test for exponentiality based on $Q_{1}$ has an extreme poor power compared to the other tests under discussion (and thus should not be recommended at all for testing $H_{0}$ ), its consistency against a large class of alternative distributions may be proved (this was conjectured by Patwardhan (1988)). The reasoning is as follows: Let $X_{1}$ have distribution function $F$ and density function $f$, where $F(0)=0$ and $E\left[X_{1}\right]=1$. From Renyi's representation of order statistics from a uniform distribution and Taylor expansion, we have

$$
\begin{aligned}
\frac{Q_{1}}{n+1} \stackrel{\underline{D}}{=} & \frac{1}{n} \sum_{j=1}^{n}(n-j+1)^{2} \\
& \cdot\left(F^{-1}\left(\frac{E_{1}+\cdots+E_{j}}{(n+1) \bar{E}_{n+1}}\right)-F^{-1}\left(\frac{E_{1}+\cdots+E_{j-1}}{(n+1) \bar{E}_{n+1}}\right)\right)^{2} \\
= & \frac{1}{n \bar{E}_{n+1}} \sum_{j=1}^{n}\left(1-\frac{j}{n+1}\right)^{2} \frac{E_{j}^{2}}{f^{2}\left(F^{-1}\left(W_{j, n}\right)\right)}
\end{aligned}
$$

where $E_{1}, \ldots, E_{n+1}$ are i.i.d. unit exponential variates with arithmetic mean $\bar{E}_{n+1}$, and

$$
\frac{E_{1}+\cdots+E_{j-1}}{(n+1) \bar{E}_{n+1}} \leq W_{j, n} \leq \frac{E_{1}+\cdots+E_{j}}{(n+1) \bar{E}_{n+1}} .
$$

The symbol " $\stackrel{\mathcal{D}}{=}$ " denotes equality in distribution. Under suitable regularity conditions on $f, Q_{1} /(n+1)$ is stochastically equivalent to

$$
\frac{1}{n} \sum_{j=1}^{n}\left(1-\frac{j}{n+1}\right)^{2} \frac{E_{j}^{2}}{f^{2}\left(F^{-1}\left(\frac{j}{n+1}\right)\right)}
$$

which converges stochastically to

$$
2 \int_{0}^{\infty} \frac{(1-F(t))^{2}}{f(t)} d t
$$

as $n \rightarrow \infty$ provided that $\int_{0}^{\infty}[(1-F(t)) / f(t)]^{4} f(t) d t<\infty$. Since the asymptotic null distribution of $n^{1 / 2}\left(Q_{1} / 2 n-1\right)$ is standard normal (see Patwardhan (1988)) 
and the quantity occuring in (4.4) has a minimum value $(=2)$ if and only if $X_{1} \sim \operatorname{Exp}(1)$ (use Jensen's inequality), the consistency of a test based on $Q_{1}$ follows.

(vi) The test of Sarkadi (Sarkadi (1975)). Sarkadi (1975) proved that a test for exponentiality rejecting the hypothesis $H_{0}$ for small values of

$$
S=\frac{\left(\sum_{i=1}^{n} \delta_{i} Y_{(i)}-n\right)^{2}}{\sum_{i=1}^{n}\left(Y_{(i)}-1\right)^{2}},
$$

where $Y_{(i)}$ and $\delta_{i}$ are given in (4.2) and (4.3) respectively, is consistent against general alternatives. Note that, apart from a constant factor, $S$ is the empirical correlation coefficient of $\left(\delta_{i}, Y_{(i)}\right), i=1, \ldots, n$. It will be seen that although being consistent, the test based on $S$ shows poor power performance and thus, should not be recommended.

Among the alternative distributions considered are the Gamma, the Weibull and the Lognormal family of distributions with scale parameter 1 and shape parameter $\theta$ as well as the Uniform, the Half-Normal, the Half-Cauchy and the $\chi_{1}^{2}$ distribution. Other families included are the Power distributions (density $\left.\theta^{-1} x^{(1-\theta) / \theta}, 0 \leq x \leq 1\right)$, the LIFR (linear increasing failure rate) distributions (density $\left.(1+\theta x) \exp \left(-\left(x+(\theta / 2) x^{2}\right)\right)\right)$ and the JSHAPE family of distributions (density $\left.(1+\theta x)^{-(\theta+1) / \theta}\right)$.

These distributions include widely used, more complex alternatives to the exponential model so as to satisfy the analyst's interest to detect the existence of such a situation. Apart from distributions with increasing and decreasing hazard rates, models with U-shaped $(\operatorname{Power}(\theta)$ for $\theta>1)$ and inverted U-shaped hazard functions $(\operatorname{Lognormal}(\theta))$ have been included. The $\operatorname{JSHAPE}(\theta)$ family has $\mathrm{J}$-shaped densities with heavier tails than the exponential distribution which arises as limiting case as $\theta \rightarrow 0$.

Estimates of powers are shown in Tables 4 and 5 . Each number represents the percentage of 5000 Monte Carlo samples declared to be significant by the various tests under discussion, rounded to the next integer. An asterisk denotes power $100 \%$. The level of significance is $5 \%$, and the sample size is $n=20$ for Table 4 and $n=50$ for Table 5 . All simulations were run on an IBM PS/2 personal computer. Using a linear congruential method to generate uniform random numbers, pseudorandom numbers of all distributions given above were generated using standard techniques (acceptance-rejection method, polar method or direct inversion).

The main conclusions that can be drawn from the simulation results are the following:

1) The tests of Patwardhan (1988) and Sarkadi (1975) have poor power over the whole range of alternatives in comparison with the other procedures under discussion and thus should not be recommended as omnibus tests for exponentiality.

2) The new test based on $T(1)$ is slightly less powerful than $M$ but slightly more powerful than both $C^{2}$ and $A^{2}$ for Gamma alternatives with $\theta>1$. In the case of these alternatives it clearly dominates $W^{*}$.

3) For Weibull alternatives with $\theta<1, T(.1)$ provides the best results, followed by $M$. In this case $T(1)$ is slightly less powerful, but comparable to $C^{2}$ and $A^{2}$. For Weibull alternatives with $\theta>1, T(1)$ is slightly better than all the other tests. 
4) For the Lognormal family, $T(1)$ is comparable in power to the omnibus tests $C^{2}$ and $A^{2}$. The same holds for LIFR distributions. For the JSHAPE family it slightly dominates over $C^{2}$ and $A^{2}$.

5) For the $\operatorname{Power}(\theta)$ family the performance of the various procedures depends markedly on the value of $\theta$.

6) Of the three new tests under discussion, $T(.1)$ provides the best results for some alternatives having infinite density at zero (Weibull $(\theta)$ for $\theta<1$, $\operatorname{Lognormal}(\theta)$ for small $\theta, \chi_{1}^{2}$ and $\operatorname{Power}(\theta)$ for large $\left.\theta\right)$. $T(10)$ works best for some alternative distributions with markedly different tail behaviour compared with the exponential distribution (Half-Cauchy, Uniform $(0,1)$ and $\operatorname{Lognormal}(\theta)$ for large $\theta$ ).

Over the whole range of alternative distributions considered, $T(1)$ constitutes a serious competitor to the omnibus tests of Cramér-von Mises and AndersonDarling, both based on the empirical distribution function.

\section{Acknowledgements}

The authors would like to thank the referees for their helpful remarks and for drawing their attention to the test of Sarkadi.

\section{REFERENCES}

Araujo, A. and Giné, E. (1980). The Central Limit Theorem for Real and Banach Valued Random Variables, Wiley, New York.

Bartholomew, D. J. (1957). Testing for departure from the exponential distribution, Biometrika, 44, 253-257.

D'Agostino, R. B. and Stephens, M. A. (1986). Goodness-of-Fit-Techniques, Dekker, New York.

Davis, Ch. S. and Stephens, M. A. (1989). Algorithm AS 248: Empirical distribution function goodness-of-fit tests, Appl. Statist., 38, 535-543.

De Wet, T, and Randles, R. H. (1987). On the effect of substituting parameter estimators in limiting $\chi^{2}, U$ and $V$ statistics, Ann. Statist., 15, 398-412.

Feller, W. (1966). An Introduction to Probability Theory and Its Applications, Vol. II, Wiley, New York.

Moran, P. A. P. (1951). The random division of an interval-Part II, J. Roy. Statist. Soc. Ser. $B, 13,147-150$.

Patwardhan, G. (1988). Tests for exponentiality, Comm. Statist. A-Theory Methods, 17, 37053722.

Sarkadi, K. (1975). The consistency of the Shapiro-Francia test, Biometrika, 62, 445-450.

Serfling, R. (1980). Approximation Theorems of Mathematical Statistics, Wiley, New York.

Shapiro, S. S. and Wilk, M. B. (1972). An analysis of variance test for the exponential distribution, Technometrics, 12, 355-370.

Shorack, G. R. (1972). The best test of exponentiality against Gamma alternatives, J. Amer. Statist. Assoc., 67, 213-214.

Spurrier, J. D. (1984). An overview of tests for exponentiality, Comm. Statist. A-Theory Methods, 13, 1635-1654.

Stephens, M. A. (1978). On the $W$ test for exponentiality with origin known, Technometrics, 20, 33-35. 